\title{
ОЦЕНКА ЭНЕРГЕТИЧЕСКИХ СПЕКТРОВ ОТРАЖЕНИЙ В ИМПУЛЬСНЫХ ДОПЛЕРОВСКИХ МЕТЕОРАДИОЛОКАТОРАХ. Ч. З. СТАТИСТИЧЕСКИЙ АНАЛИЗ МЕТОДОВ ВОСПРОИЗВЕДЕНИЯ НЕПРЕРЫВНЫХ СПЕКТРОВ ОТРАЖЕНИЙ ОТ МЕТЕООБРАЗОВАНИЙ
}

\author{
Д. И. ЛЕХОВИЦКИЙ ${ }^{1}$, Д. В. АТАМАНСКИЙ ${ }^{2}$, Д. С. РАЧКОВ ${ }^{1}$, А. В. СЕМЕНЯКА ${ }^{1}$ \\ ${ }^{1}$ Харьковский национальный университет радиоэлектроники, \\ Украина, Харьков, 61166, пр-т Ленина, 14 \\ ${ }^{2}$ Харьковский университет Воздушных Сил имени Ивана Кожедуба, \\ Украина, Харьков, 61023, ул. Сумская 77/79
}

\begin{abstract}
Аннотация. Обоснована методика, приведены количественные результаты аналитического и экспериментального исследования и сравнения статистических характеристик классических и «параметрических» методов воспроизведения энергетических спектров междупериодных флуктуаций отражений различной природы (в том числе от метеообразований) в импульсных РЛС. Исследована типичная для практики «адаптивная» ситуация, в которой априори неизвестная корреляционная матрица отражений заменяется оценками различного вида, формируемыми по обучающим выборкам конечного объема. Обоснованы алгоритмы, целесообразные для использования в импульсных РЛС различного назначения, в том числе в импульсных доплеровских метеорадиолокаторах. Обсуждаются рациональные способы их практической реализации на унифицированной основе адаптивных решетчатых фильтров.
\end{abstract}

Ключевые слова: процесс авторегрессии; энергетический спектр; методы спектрального оценивания; спектральная функция; адаптивный решетчатый фильтр; корреляционная матрица; оценка максимального правдоподобия; метод максимальной энтропии Берга; метод минимальной дисперсии Кейпона

\section{ВВЕДЕНИЕ И ПОСТАНОВКА ЗАДАЧИ}

Статья посвящена сравнению качества воспроизведения энергетических спектров междупериодных флуктуаций отражений от метеообразований (MO) известными и новыми методами спектрального оценивания (CO) в реальных условиях использования вместо априори неизвестной корреляционной матрицы $(\mathrm{KM)} \mathrm{анализируемой} \mathrm{смеси} \mathrm{ее} \mathrm{оценок} \mathrm{различ-}$ ного вида, формируемых по обучающим выборкам конечного объема. Она продолжает статьи $[1,2]$ и содержит 2 раздела и заключение.

В первом разделе описана методика сравнения методов СО и сравниваются возможно- сти воспроизведения на их основе спектров МО в указанных условиях. В роли показателей качества выступают средние значения $\overline{\widehat{\varepsilon}}_{1}$ и $\overline{\widehat{\varepsilon}}_{2}$ случайных в этих условиях критериев «интегральной относительной среднеквадратической ошибки» (ПИК) [1.30] и «интегральной относительной ошибки» (ВИК) [2.3] соответственно.

Для большинства методов и используемых оценок КМ эти показатели определяются по результатам математического моделирования, хотя для некоторых из них удается получить достаточно точные аналитические приближения к искомым средним значениям $\overline{\bar{\varepsilon}}_{1}$ ПИК и 
Оиенка энергетических спектров отражений в импульсных доплеровских метеорадиолокаторах. 4. 3. Статистический анализ методов воспроизведения непрерывных спектров отражений от метеообразований

\section{БИБЛИОГРАФИЧЕСКИЙ СПИСОК}

1. Оценка энергетических спектров отражений в импульсных доплеровских метеорадиолокаторах. Ч. 1. Разновидности алгоритмов спектрального оценивания / Д. И. Леховицкий, Д. В. Атаманский, Д. С.Рачков, А.В.Семеняка // Известия вузов. Радиоэлектроника. - 2015. - T. 58, № 12. - C. 3-30. - DOI : $10.20535 / \mathrm{S} 0021347015120018$.

2. Оценка энергетических спектров отражений в импульсных доплеровских метеорадиолокаторах. Ч. 2. Предельные возможности / Д.И. Леховицкий, Д.В. Атаманский, Д.С. Рачков, А.В. Семеняка // Известия вузов. Радиоэлектроника. — 2016. - Т. 59. № 9. - C. 3-20. - DOI : 10.20535/S0021347016090016.

3. Reed, I. S. Rapid convergence rate in adaptive arrays / I. S. Reed, J. D. Mallett, L. E. Brennan // IEEE IEEE Trans. Aerosp. Electron. Syst. - Nov. 1974. Vol. AES-10, No. 6. - P. 853-863. - DOI : 10.1109/TAES.1974.307893.

4. Абрамович, Ю.И. Анализ эффективности адаптивной максимизации отношения сигнал/помеха, использующей обращение оценки корреляционной матрицы / Ю.И. Абрамович, А.И.Неврев // Радиотехника и электроника. - 1981. - Т. 26, № 12. C. $2558-2566$.

5. Abramovich, Y. I. Band-inverse TVAR covariance matrix estimation for adaptive detection / Yuri I. Abramovich, Nicholas K. Spencer, Ben A. Johnson // IEEE Trans. Aerosp. Electron. Syst. — Jan. 2010. Vol. 46, No. 1. - P. 375-396. - DOI : 10.1109/TAES.2010.5417169.

6. Adaptive lattice filters for band-inverse (TAVR) covariance matrix approximations / D. Lekhovytskiy, Y. Abramovich / Int. Radar Symp. IRS2009. - 09-11
Sept. 2009, Hamburg, Germany : proc. - Hamburg, 2009. - P. 535-539.

7. Ленточно-диагональная регуляризация МП оценок корреляционных матриц гауссовых помех в алгоритмах адаптации антенных решеток / Д. И. Леховицкий, Ю.И.Абрамович, Г.А.Жуга, Д.С.Рачков // Прикладная радиоэлектроника. - 2010. — Т. 9, № 1 . - C. 107-121.

8. Abramovich, Y. I. A Modified GLRT and AMF framework for adaptive detectors / Yuri I. Abramovich, Nicholas K. Spencer, Alexei Y. Gorokhov // IEEE Trans. Aerosp. Electron. Syst. — Jul. 2007. — Vol. 43, No. 3. P. 1017-1051. - DOI : 10.1109/TAES.2007.4383590.

9. Burg, J. P. A New Analysis Technique for Time Series Data / J. P. Burg // Modern Spectrum Analysis. IEEE Press, 1978 [ed. by D. G. Childers]. - P. 42-48.

10. Фридландер, Б. Решетчатые фильтры для адаптивной обработки данных / Б.Фридландер // ТИИЭР. - 1982. — Т. 70, № 58. - С. 54-97.

11. Фридландер, Б. Методы спектрального оценивания на основе решетчатой структуры / Б. Фридландер // ТИИЭР. - 1982. — Т. 70, № 9. - С. 95-125.

12. Адаптивные фильтры / Под ред. К.Ф.Н.Коуэна и П. М. Гранта ; пер. с англ. — М. : Мир, 1988. $392 \mathrm{c}$.

13. Semeniaka, A. V. Comparative analysis of Toeplitz covariance matrix estimation methods for space-time adaptive signal processing / A. V. Semeniaka, D. I. Lekhovytskiy, D. S. Rachkov // Proc. of IEEE CIE Int. Conf. on Radar : RADAR-2011, 24-27 Oct. 2011, Chengdu, China : proc. — IEEE, 2011. - P. 696-699. DOI : $10.1109 /$ CIE-Radar.2011.6159636.

14. Хастингс, Н. Справочник по статистическим распределениям / Н. Хастингс, Дж. Пикок. - М. : Статистика, 1980. - 95 с.

15. Nitzberg, R. Application of maximum likelihood estimation of persymmetric covariance matrices to adaptive processing / Ramon Nitzberg // IEEE Trans. Aerosp. Electron. Syst. - Jan. 1980. - Vol. AES-16, No. 1. P. 124-127. - DOI : 10.1109/TAES.1980.308887.

16. Абрамович, Ю. И. Регуляризованный метод адаптивной оптимизации по критерию максимума отношения сигнал/помеха / Ю.И.Абрамович // Радиотехника и электроника. - 1981. - Т. 26, № 3. C. 543-551.

17. Леховиикий, Д. И. Методы адаптивной решетчатой фильтрации в задачах пространственно-временной обработки сигналов / Д.И.Леховицкий, В.И.Зарицкий, И.Д.Раков, [и др.]. - М. : РТИ АН СССР, 1987. - 30 с. - (Препринт / АН СССР, РТИ, № 8610, 1987).

18. Леховицкий, Д. И. О стратегии использования МП оценок КМ в многоканальных системах адаптивной обработки сигналов на фоне помех / Д.И. Леховицкий, С.В.Полишко, Г.А.Жуга // Глобальные информационные системы. Проблемы и тенденции развития : 1-я междунар. научная конф., 2006 : тезисы докл. - Харьков-Туапсе, 2006. - С. 444-445. 
19. СДЦ в импульсных РЛС: 3. Структуры, параметры и эффективность квазиоптимальных систем междупериодной обработки гауссовых сигналов на фоне гауссовых пассивных помех / Д.И.Леховицкий, В. П.Рябуха, Г. А. Жуга, Д. С. Рачков // Прикладная радиоэлектроника. - 2009. - Т. 8, № 2. - С. 109-123.

20. Экспериментальные исследования систем СДЦ на основе адаптивных решетчатых фильтров в импульсных РЛС с попачечной вобуляцией периодов зондирования / Д.И.Леховицкий, В.П.Рябуха, Г. А. Жуга, В.Н. Лаврентьев // Прикладная радиоэлектроника. - 2008. - Т. 7, № 1. - С. 3-16.

21. Стратонович, Р. Л. Принципы адаптивного приема / Р. Л. Стратонович. - М. : Сов. радио, 1973. $144 \mathrm{c}$.

22. Abramovich, Y. I. Order estimation and discrimination between stationary and time-varying (TVAR) autoregressive models / Yuri I. Abramovich, Nicholas K. Spencer, Michael D. E. Turley // IEEE Trans. Signal Process. - Jun. 2007. - Vol. 55, No. 6. P. 2861-2876. — DOI : 10.1109/TSP.2007.893966.

23. Уидроу, Б. Адаптивная обработка сигналов / Б. Уидроу, С. Стирнз : пер. с англ. - М. : Радио и связь, 1989. - $440 \mathrm{c}$.

24. Монзинго, Р. А. Адаптивные антенные решетки: Введение в теорию / Р. А. Монзинго, Т. У. Миллер : пер. с англ. - М. : Радио и связь, 1986. — 448 с.

25. Кокин, В. Н. Использование текущей оценки обратной корреляционной матрицы помех в адаптивном обнаружителе / В.Н.Кокин, А.В.Темеров, В. В. Фединин // Радиотехника и электроника. - 1980. — T. 25, № 7. - C. 1540-1542.

26. Пат. № 3876847 США, МКИ $325 / 367$ от 8.04.1975 / С. Гираудон.

27. Лексаченко, В. А. Синтез многомерного «выбеливающего» фильтра по методу Грама-Шмидта / В.А.Лексаченко, А.А.Шаталов // Радиотехника и электроника. - 1976. - Т. 21, № 1. - С. 112-119.

28. Бондаренко, Б. Ф. Применение методов функционального анализа для решения задач синтеза системы пространственно-временной обработки сигналов / Б. Ф. Бондаренко, В. П. Прокофьев // Известия вузов. Радиоэлектроника. - 1982. - Т. 25 , № 7 . C. $12-16$.

29. Ширман, Я. Д. Теория и техника обработки радиолокационной информации на фоне помех / Я.Д.Ширман, В.Н.Манжос. - М. : Радио и связь, 1981. - $416 \mathrm{c}$.

30. Спектральный анализ отражений в доплеровских метеорадиолокаторах / Д.И.Леховицкий, Д.В.Атаманский, Д.С.Рачков, А.В.Семеняка // Радиолокация, навигация, связь : XVII междунар. науч.-техн. конф., 12-14 апр. 2011, Воронеж, РФ. Воронеж, 2011. - С. 1968-1979.

31. СДЦ в импульсных РЛС: 5. Адаптивные системы междупериодной обработки гауссовых сигналов на фоне гауссовых пассивных помех / Д.И. Леховицкий, В.П.Рябуха, Г.А.Жуга, Д.С.Рачков, А.В.Семе- няка // Прикладная радиоэлектроника. — 2011. T. 10 , № 4. - C. 506-525.

32. Efremov, $V$. Results of theoretical and experimental investigations of meteorological formation power spectrum using «superresolution» methods / V. Efremov, V. Laurukevich, D. Lekhovytsky, I. Vylegzhanin, B. Vovshin // Int. Radar Symp. : IRS2009, 09-11 Sept. 2009, Hamburg, Germany : proc. - Hamburg, 2009. - P. 777-784.

33. Laurukevich, $V$. Estimation of energy, spectral and polarimetric characteristics of meteorological echoes in DMRL-C / V. Laurukevich, A. Pushkov, I. Vylegzhanin, et al. // Int. Radar Symp. : IRS2011, 7-9 Sept. 2011, Leipzig, Germany : proc. — Leipzig, 2011. — P. 267-272.

34. Efremov, $V$. The new generation of Russian C-band meteorological radars. Technical features, operation modes and algorithms / V. Efremov, I. Vylegzhanin, B. Vovshin // Int. Radar Symp. : IRS2011, 7-9 Sept. 2011, Leipzig, Germany : proc. — Leipzig, 2011. P. 239-244.

35. Lekhovytskiy, D. I. Statistical analysis of estimation accuracy of the meteorological formations parameters in pulsed Doppler weather radars with arbitrary staggering of pulse repetition intervals / D. I. Lekhovytskiy, D. S. Rachkov, A. V. Semeniaka, et al. // Int. Radar Symp. : IRS2011, 7-9 Sept. 2011, Leipzig, Germany : proc. Leipzig, 2011. - P. 273-278.

36. Laurukevich, $V$. The results of field tests of russian meteorological radar "DMRL-C" / V. Laurukevich, A. Pushkov, B. Vovshin, I. Vylegzhanin // IV международный радиоэлектронный Форум : 18-21 окт. 2011, Харьков, Украина : труды конф. - Харьков, 2011. T. 1. - C. 7-12.

37. Берг, Дж. П. Оценивание ковариационных матриц с заданной структурой / Дж. П. Берг, Д. Г. Люнбергер, Д. Л. Венгер // ТИИЭР. — 1982. — Т. 70, № 9. - C. 63-77.

38. Адаптивные решетчатые фильтры. Часть I. Теория решетчатых структур / Д.И.Леховицкий, Д.С.Рачков, А.В.Семеняка, В.П.Рябуха, Д.В.Атаманский // Прикладная радиоэлектроника. - 2011. T. 10, № 4. - С. 380-404.

39. Адаптивные решетчатые фильтры. Часть II. Алгоритмы настройки АРФ / Д.И.Леховицкий, Д.С.Рачков, А.В.Семеняка, В.П.Рябуха, Д.В.Атаманский // Прикладная радиоэлектроника. - 2011. T. 10, № 4. - С. 405-418.

40. Повышение точности однозначного измерения скорости метеообразований в доплеровских метеолокаторах с вобуляцией интервалов зондирования / Д.И.Леховицкий, Д.В.Атаманский, Д.С.Рачков, А.В.Семеняка // Известия вузов. Радиоэлектроника. - 2015. - T. 58, № 9. - C. 3-22. - DOI : 10.20535/S0021347015090010.

41. Lekhovytskiy, D. I. To the theory of adaptive signal processing in systems with centrally symmetric receive channels / D. I. Lekhovytskiy // EURASIP J. Advances 
Signal Process. - 2016. - Vol. 33. - P. 1-11. — DOI : 10.1186/s13634-016-0329-z.

42. Сравнение эффективности адаптивной обработки в произвольных и центрально-симметричных ФАР / Д.И. Леховицкий, Д.В.Атаманский, И.Г.Кириллов, В.И. Зарицкий // Антенны. - 2000. - № 1. C. $30-35$.

43. Рябуха, В. П. Адаптивные системы защиты РЛС от шумовых помех. Часть І. Корреляционные автокомпенсаторы на основе стохастических градиентных алгоритмов адаптации / В.П.Рябуха // Прикладная радиоэлектроника. - 2016. - Т. 15, № 1. C. 11-25.

44. Lekhovytskiy, D. I. Thirty years experience in development of adaptive lattice filters theory, techniques and testing in Kharkiv / D. I. Lekhovytskiy // VIII Int. Conf. on Antenna Theory and Techniques : ICATT2011, 20-23 Sept. 2011, Kyiv, Ukraine : proc. - IEEE, 2011. P. 51-56. - DOI : 10.1109/ICATT.2011.6170713.

45. Sayed, A. H. Fundamentals of adaptive filtering / A. H. Sayed. - NJ, Hoboken : John Wiley and Sons Inc., 2003. - $1125 \mathrm{p}$.

46. Джиган, В. И. Адаптивная фильтрация сигналов: теория и алгоритмы / В. И. Джиган. - М. : Техноcфера, 2013. - 528 c.

47. Lattice implementation of "superresolving" methods for meteorological objects spectra estimation / Dmytro S. Rachkov, David I. Lekhovytskiy, Andrii V. Semeniaka, Boris M. Vovshin, Uladzimir U. Laurukevich // 15th Int. Radar Symp. : IRS 2014, 16-18 June 2014, Gdansk, Poland : proc. - IEEE, 2014. - P. 35-38. DOI : 10.1109/IRS.2014.6869229.

48. Rachkov, D. S. Lattice-filter-based unified structure of system for interperiod processing of weather radar signals / Dmytro S. Rachkov, David I. Lekhovytskiy // IEEE Int. Radar Conf. : 10-15 May 2015, Arlington, USA : proc. - IEEE, 2015. - P. 1234-1239. - DOI : 10.1109/RADAR.2015.7131183.

49. Lekhovytskiy, D. I. K-rank modification of adaptive lattice filter parameters / David I. Lekhovytskiy, Dmytro S. Rachkov, Andrii V. Semeniaka // IEEE Int. Radar Conf. : 10-15 May 2015, Arlington, USA : proc. IEEE, 2015. - P. 127-132. - DOI : 10.1109/RADAR.2015.7130983.

50. Рекуррентные алгоритмы настройки адаптивных решетчатых фильтров / Д.И. Леховицкий, В.П.Рябуха, Д.С.Рачков, А.В. Семеняка // ТКЭА. 2016. - № 2-3. - C. 26-32. - DOI : 10.15222/TKEA2016.2-3.26.

51. Lev-Ari, H. Schur and Levinson algorithms for nonstationary processes / H. Lev-Ari, T. Kailath // Acoustic, Speech and Signal Processing : IEEE Int. Conf. ICASSP, 30 Mar.-1 Apr. 1981, Atlanta, CA, USA : proc. - IEEE, 1981. - P. 860-864. - DOI : 10.1109/ICASSP.1981.1171194.

52. Sharman, K. C. Spatial lattice filter for high-resolution spectral analysis of array data / K. C. Sharman, T. S. Durrani // IEE Proc. F: Commun. Radar Signal Process. - Apr. 1983. - Vol. 130, No. 3. - P. 279-287. — DOI : 10.1049/ip-f-1:19830047.

53. Lattice-filter-based ground clutter canceller for pulse Doppler weather radar / Dmytro S. Rachkov, David I. Lekhovytskiy, Andrii V. Semeniaka, Viacheslav P. Riabukha, Dmytro V. Atamanskiy // 15th Int. Radar Symp. : IRS 2014, 16-18 June 2014, Gdansk, Poland : proc. - IEEE, 2014. - P. 215-219. - DOI : 10.1109/IRS.2014.6869251. 